\title{
Experiencias en la intervención psicológica con familias de personas dependientes
}

\section{Psychological intervention with families of dependent people}

Maria Dolores ORTIZ MUÑOZ*

\section{RESUMEN}

La atención e intervención con familias es fundamental para realizar programas de intervención eficaces con personas dependientes. Entre otras disciplinas, la psicología y sus profesionales han realizado numerosas contribuciones a nivel teórico y y práctico con este colectivo. Existen numerosas necesidades de atención y programas de intervención psicológicos con familiares cuidadores. En este artículo se presenta un ejemplo de intervención con familiares de personas mayores dependientes.

\section{PALABRAS CLAVE}

Dependencia, Cuidadores informales, Cuidadores familiares, Programas de intervención psicológicos con familias.

\section{ABSTRACT}

The intervention with families is compulsory in the assistance to people with dependences, all this is important to carry out efficient intervention programs. Among other disciplines, Psychology and its professionals have carried out some contributions in practice and in theory referring to this group of people. All this has to do with assistance needs and psychological intervention programs. Examples of practical interventions with families of elderly dependent people are shown in this article.

\section{KEY WORDS}

Dependence, Informal caregivers, Family caregivers, Intervention programs psychological with families.

\footnotetext{
* Psicóloga privada experta en gerontología.
} 


\section{PSICOLOGÍA E INTERVENCIÓN CON FAMILIAS DE PERSONAS DEPENDIENTES}

El concepto de dependencia fue definido por el Consejo de Europa como un estado en el que se encuentran las personas que, como consecuencia de una falta o pérdida de autonomía fisica, psíquica o social, tienen la necesidad de asistencia y/o ayudas importantes a fin de realizar los comportamientos habituales de la vida diaria. El apoyo a las familias con un miembro en situación de dependencia se ha convertido en una necesidad prioritaria en nuestra sociedad. Existen numerosos factores demográficos, sociales, políticos, económicos que justifican esta prioridad, entre ellos se destacan dos: 1) El aumento de las necesidades de atención a personas dependientes (aumento en número y prolongación del tiempo de cuidados). 2) La disminución de capacidad de atención familiar, institución tradicionalmente cuidadora en nuestro país (crisis del apoyo informal).

Con la aprobación de la "Ley de Promoción de la Autonomía personal y Atención a las personas en situación de Dependencia" se pondrá en marcha el sistema de protección social a la dependencia, donde se deberán complementar e integrar los recursos formales e informales (Santo, M., Rodríguez, P., 2002). Algunos autores especializados (Rodríguez P., 2005) recomiendan que las familias se tengan en cuenta tanto la hora de evaluar la dependencia, como en el diseño de los Programas Individualizados de Atención así como incorporar servicios de respiro y apoyo familiar a la cartera de servicios. La atención a la familia y más en concreto a los cuidadores familiares se convierten en piedra angular del adecuado abordaje de la atención a las personas dependientes.

A nivel Profesional, la atención a las personas dependientes comenzó centrándose exclusivamente en la persona en situación de dependencia (sentimientos, problemas, necesidades,...), poco a poco, los modelos han ido evolucionando y ampliando el interés de intervención hacia los cuidadores principales, para luego incluir a otros miembros de la familia, profesionales y a la sociedad en la que vive. La intervención con familias de personas dependientes tiene carácter en sí misma interdisciplinar. La psicología cuenta con un cuerpo de conocimientos que junto con instrumentos y técnicas psicológicas avaladas experimental y científicamente capacita a sus profesionales para intervenir eficazmente. Revisando la base de datos psicológica psiocodoc (agosto 2006), existen 3992 referencias sobre familias, referencias que comienzan a aparecer en la década de los 80 aumentando progresivamente hasta hoy en día.

Centrándonos en el cuidado, la psicologia ha aportado varios modelos teóricos. Los primeros modelos e intervenciones parten de salud mental comunitaria $\mathrm{y}$ pronto se generalizan a otros colectivos. Entre los modelos del cuidado y atendiendo a una revisón realizada por Martínez F., Villalba C. Y García M. (2001), se destacan los siguientes autores. La aportación más clásica y básica la realiza Zarit (1980), manteniendo que una situación prolongada de cuidado amenaza la salud física y mental del familiar que asume la responsabilidad, es lo que conocemos como "carga de cuidados". A nivel teórico, Perlin (1990) realiza una conceptualización en la que el paradigma clásico de estrés se aplica al campo de los cuidadores informales. El cuidado sería considerado como paradigma de situación estresora crónica. Surgen así mismo nuevos modelos teóricos que contemplan la acción de cuidar en términos de dimensiones múltiples (Lawton, M. P., Brody, E. M., Saperstein, 
1989), (Romeis, 1989). Kahana (1994) ofrece un paradigma de desarrollo de cuidados integrando en la acción de cuidar dimensiones personales, sociales y espacio-temporales (quién, cuándo, qué procesos se producen e interacciones entre los tres). Braithwait (1996) incorpora la percepción de la relación entre cuidadores y familiares dependientes, considerando que el estrés se produciría dentro de la dinámica social y dando importancia a lo que llama "crisis de declive" entendida esta como las amenazas de la relación entre cuidador y familiar dependiente derivadas de las normas culturales y expectativas de cuidado. Otros modelos de estrés y afrontamiento clásicos utilizados son el de Lazarus y Folieman (1984) que han sido adaptados a la situación de cuidado (Haley, W. E., Levise, E. G., Brown, S. L., Berry, J. W., y Hughes, G. H., 1987), (Vitaliano et al. 1991) donde se tienen en cuenta variables de contexto (demandas de cuidado, grado de percepción, evaluación del cuidador de las demandas de cuidado como estresantes), variables mediadoras (estrategias de afrontamiento / apoyo social) y consecuencias de las demandas de cuidado (físicas, emocionales).

No se trata de psicologizar ni de dar una visión problemática y patológica de las personas con dependencia y de sus familias. El principio de normalidad debe regir las intervenciones, la familia con una persona dependiente en su seno no debe diferir en principio de otra familia que no tenga personas dependientes a su cargo (Martínez, et. al., 2001). Se trata de intervenir con las familias para que éstas sean más eficaces y capaces de asumir durante un periodo prolongado de tiempo el cuidado responsable de su familiar (con apoyos o sin apoyos), facilitando su adaptación, reduciendo su vulnerabilidad, potenciando sus recursos y detectando casos que requieran atención especializada.

\section{EVALUACIÓN PRELIMINAR: IDENTIFICACIÓN DE NECESIDADES DE ATENCIÓN / INTERVENCIÓN PSICOLÓGICAS}

El primer paso en la planificación de la intervención consiste en conocer las características de la familia cuidadora, sus necesidades concretas y/ o problemas prioritarios. Este conocimiento identifica las necesidades concretas de intervención psicológica familiar. En este sentido, destacar la investigación sobre cuidadores de personas mayores publicada por el INSERSO (1995) y repetida una década mas tarde por el IMSERSO (2005).

La identificación de necesidades y demandas de atención / intervención psicológicas podemos realizarlas a través de: 1) Evaluaciones integrales de la situación de dependencia, 2) Evaluaciones psicológicas sobre aspectos específicos, 3) Revisión de historias clínicosociales, 4) Revisión biobliográfica y de otras experiencias, 5) Entrevistas familiares, y/o profesionales etc.

A continuación se presentan algunas necesidades de intervención psicológica con familiares y cuidadores principales. La exposición se organiza diferenciando dos fuentes principales, 1) necesidades detectadas por los profesionales y 2) necesidades expresadas por los familiares.

\section{Necesidades detectadas por los profesionales}

\section{Necesidades de atención familiar: Necesidades de la persona dependiente}

Cualquier necesidad detectada en la persona en situación de dependencia puede ser objeto de intervención familiar 
/ intervención psicológica familiar. Sobre este aspecto resulta fundamental la intervención con las familias, por el papel principal que ésta desarrolla, como amortiguadora del impacto que la discapacidad o enfermedad incapacitante tiene sobre la persona, como facilitadora del proceso de adaptación a la dependencia, como proveedora de cuidados y apoyo, cómo promotora de salud, bienestar, autonomía y autoestima, como facilitadora de la rehabilitación/habilitación del déficit funcional recuperable, como facilitadora de desarrollo cognitivo, afectivo, social y de personalidad en los niños / adolescentes con dependencia, como herramienta básica para el manejo y reducción (frecuencia, duración e intensidad) de las alteraciones de comportamiento etc.. Destacar que existen actitudes familiares que van a facilitar y otras que van a dificultar la atención y promoción de autonomía en la persona en situación de dependencia (Ej Sobreprotección; actitudes negativas frente a la discapacidad y la persona discapacitada, envejecimiento y las personas mayores, enfermedad y las personas con enfermedad mental, crónica o terminal).

Las necesidades de atención van a variar en función de múltiples factores, algunos de ellos son:

1) Momento evolutivo que se encuentre la persona (recién nacido, infancia, adolescencia, edad adulta, vejez, enfrentamiento a la muerte)

2) Características específicas de la situación o enfermedad que ha generado la dependencia: congénita, hereditaria, adquirida (enfermedad, accidente, atentado terrorista...), enfermedad terminal, etc.

3) Curso y evolución de la situación de dependencia. Dependencia temporal, dependencia permanente e irreversible, dependencia en aumento, grado de dependencia variable (periodos asintomáticos / periodos sintomáticos), dependencia como consecuencia de una enfermedad en fase terminal, etc

4) Cantidad de áreas afectadas y severidad de afectación de la persona dependiente.

5) Impacto de la dependencia en la persona y el nivel adaptación / aceptación.

6) Presencia /ausencia alteraciones de comportamiento.

7) Presencia / ausencia de psicopatología asociada a la situación de dependencia.

8) Capacidad / nivel de conciencia que la persona dependiente tiene de su situación

9) Nivel de desarrollo cognitivo/social.

10) Otras variables individuales: Rasgos de personalidad, vulnerabilidad al estrés, resistencias al cambio, actitudes, rol antes /después de la situación de dependencia, situación económica individual y/o familiar etc.

\section{Necesidades de atención: La familia en si misma}

La familia tiene sus propias necesidades de atención derivadas de tener en su seno un familiar dependiente.

- Necesidades de Información, orientación y formación: 1) En cuanto al curso y progresión de la enfermedad 
o características y consecuencias de la situación que origina la dependencia. 2) habilidades y destrezas para realizar tareas de cuidado y autocuidado, manejo de problemas de conducta, situaciones dificiles y de momentos críticos. 3) La utilización de recursos y ayudas técnicas existentes en la comunidad.

- Necesidades de apoyo emocional derivadas de tener un familiar en situación de dependencia. Necesidad de descarga afectiva y expresión emocional del dolor y sufrimiento que supone tener un ser querido en situación de dependencia.

- Necesidades surgidas durante el proceso de adaptación de los cuidadores familiares a la situación de dependencia y a los diferentes momentos criticos surgidos durante el proceso de cuidados. La atención de la dependencia es un proceso temporalmente extenso que abarca desde el diagnóstico/comunicación de la situación que produce dependencia hasta la atención del familiar tras el fallecimiento de la persona dependiente. El conocimiento previo de qué situaciones criticas se darán en el proceso de cuidado, puede ser muy útil para planificar intervenciones familiares antes, durante y después de que éstas se produzcan.

- Necesidades derivadas de la evaluación del estrés originado por asumir el cuidado. Incluye la valoración de la carga (objetiva /subjetiva), estresores presentes y estrategias de afrontamiento, así cómo el estudio de variables de personalidad que actuarian como mediadoras en el proceso de estrés y las consecuencias sobre la salud de los cuidadores.
- Necesidades surgidas por la presencia de otros acontecimientos vitales estresantes.

- Necesidades surgidas de la estructura y funcionamiento familiar. Analizar funcionamiento familiar previo y posterior a la situación de dependencia o momento crítico. Destacar la flexibilidad de roles como facilitador de adaptación.

- Necesidades surgidas de compaginar el cuidado con los planes vitales de todos los miembros de la familia.

- Necesidades derivadas de la historia familiar, de la discapacidad enfermedad incapacitante o terminal.

- Necesidades derivadas de la detección en los cuidadores de consecuencias negativas derivadas de asumir el cuidado de su familiar: conflictos familiares, reacciones emocionales, empeoramiento de la salud, repercusiones a nivel laboral y económico, reducción o ausencia de actividades de ocio.(Izal M., Montorio I., Díaz P., 1997).

- Necesidades que pueden presentar los individuos del sistema familiar ante el padecimiento de una enfermedad en la fase final de la vida por algún miembro de la familia: agotamiento físico y mental, labilidad emocional, depresión, trastornos de ansiedad especialmente miedos, problemas de consumo abusivo (alcohol, tabaco, comida, compras, etc.), trastornos del sueño (insomnio, pesadillas...), alteraciones del apetito y del peso, síntomas hipocondriacos, reacciones fóbicas, conducta dependiente, problemas laborales, regresión conductual (ej. En niños falta de control de esfinteres), problemas de aprendizaje, concen- 
Experiencias en la intervención con familias de personas dependientes

tración, escolaridad y conducta (Arranz P., Barbero J, Barreto P. Y Bayés R., 2003).

- Necesidades de atención especilizada de salud mental en cuidadores familiares. Saber derivar en caso de duelos complicados, cuadros de ansiedad y depresión clinicamente significativos, riesgo de suicidio, o presencia de otras psicopatologias.

- Necesidades surgidas de la detección de situaciones de violencia o malos tratos: A veces la víctima es la persona dependiente (ej. Inversión rol victima / maltratador, sobrecarga del cuidador, etc.), en otras ocasiones la víctima son los cuidadores familiares (ej. Cuidador mayor de un adulto con problemas de salud mental).

- Necesidades surgidas de la evaluación de recursos familiares: educación, habilidades personales de afrontamiento (Capacidad de solución de problemas, habilidades sociales / comunicación, petición ayuda,...),situación económica..

- Necesidades surgidas de la evaluación del apoyo social: cantidad y calidad de las relaciones, apoyo espiritual, red de relaciones sociales.

- Necesidades de atención: diferencias de género. $\mathrm{El}$ rol de cuidador familiar suele tener género femenino y número singular. Necesitamos promover un mayor compromiso masculino en el cuidado (Rodríguez P., 2005). Las estrategias de intervención variarán en función del género: diferencias en motivación, formas de desempeño de rol, estilos de afrontamiento y psicopatologia asociada (Martínez, et. al, 2001).
- Necesidades de atención por envejecimiento del cuidador principal.

- Necesidades de atención: Relación de parentesco / otros miembros de la familia/ grupo referencia. La relación de parentesco entre la persona cuidada y el cuidador principal genera diferentes tipologias de cuidadores principales "padres", "hijos", "cónyuges", con caracteristicas propias de intervención grupal. El resto de miembros familiares también padecen consecuencias derivadas del cuidado, siendo figuras claves para asegurar la atención adecuada y sostenida en el tiempo de la persona dependiente, y para que el cuidador principal tenga apoyo y respiro. Otros miembros susceptibles de intervención son los miembros del grupo de referencia (amigos, compañeros de trabajo o de ocio, etc.).

- Necesidades derivadas de las características psicológicas de cada uno de los miembros de la unidad familiar. Rasgos de personalidad, actitudes, vulnerabilidad al estrés, ideas distorsionadas, ...

- Necesidades de atención: estigma social. Podemos encontrarnos con frecuencia situaciones que favorecen el estigma social de la persona dependiente /familia. Consecuencias de este estigma social son entre otras el aislamiento social, dificultades a nivel de integración educativa, formativa, de integración laboral 0 de acceso a las actividades de ocio.

- Otras necesidades de atención: Destacar las provenientes del contexto de cuidado(casa, hospital, centro residencial.../rural-urbano) la disponibilidad de servicios y recursos así como la importancia de los factores culturales en una sociedad que 
avanza hacia la multiculturalidad (población emigrante con discapacidad / cuidadora).

\section{Necesidades de atención: Relación familia / profesionales-recursos formales}

Son destacables: 1)Necesidades surgidas por las resistencias, barreras psicológicas a aceptar, solicitar o utilizar recursos adecuados o delegar cuidado; 2) Necesidades surgidas por problemas de adaptación de familias al funcionamiento de los centros/servicios; 3) Necesidades surgidas por falta de implicación familiar 0 claudicación en los cuidados; 4) Necesidades surgidas por ignorar los profesionales / servicios, las preferencias y prioridades de atención que expresan los familiares / las personas dependientes; 5) Necesidades surgidas por errores cometidos por profesionales / centros / servicios.

\section{NECESIDADES EXPRESADAS POR LOS FAMILIARES}

Debemos de detectar que necesidades sienten que tienen los familiares, asi como el orden de prioridad que establecen. Dar respuesta a estas necesidades asegura una mayor eficacia y adhesión a la intervención que realicemos.

\section{Ejemplos de evaluación psicológica}

Evaluación Psicológica del Cuidador Principal (personas mayores dependientes). (Tabla 1)

Evaluación Psicológica de las familias ( hijos con deficiencia visual adquirida). (Tabla 2)

Existe una amplia gama de programas $e$ intervenciones psicológicas con familias dependiendo de las diferentes necesidades que se quieran cubrir, perfil de la persona en situación de dependencia, áreas en las que se quiera intervenir y el contexto en el que se quiera intervenir. Tras una evaluación preliminar para identificar las características, necesidades y problemas concretos que atañen a la familia, deberemos de plantear las intervenciones de forma realista definiendo lo que vamos a hacer en función de la interrelación entre lo que se debe hacer, lo que queremos hacer y lo que podemos hacer. Una vez decidido el tipo y modelo de intervención fijaremos los objetivos generales y específicos. En la intervención con familias pueden, y algunos casos deben de estar implicados profesionales de diferentes disciplinas sociales, sanitarias, etc., según el programa de intervención exija la incorporación o no, de otros perfiles profesionales.

\section{PROGRAMAS DE INTERVENCIÓN PSICOLÓGICA CON FAMILIAS DE PERSONAS DEPENDIENTES}

¿Qué objetivos generales son más frecuentes? 1) Mejorar el cuidado y atención de persona dependiente por parte su familia. 2) Reducir niveles de estrés derivados de la situación de cuidado. 3) Facilitar el proceso de adaptación familiar a la dependencia reduciendo vulnerabilidad y fomentando recursos.

¿A quienes van dirigidos Programas Intervención Psicológicos? Principalmente se dirigen hacia los cuidadores principales / familiares involucrados cuidado ( hermanos, abuelos, cónyuges, hijos, nietos,..) y miembros del grupo de referencia.

¿Cuándo intervenir? Cuanto antes mejor y durante todo el proceso de cuidado especialmente cuando existan situaciones o momentos críticos. 
Experiencias en la intervención con familias de personas dependientes

Tabla 1. Valoración del cuidador

(Leturia, Yanguas, Arriola, Uriarte, 2001)

\begin{tabular}{|c|c|}
\hline ÁREAS A VALORAR & OBJETIVOS DE LA VALORACIÓN \\
\hline Contexto del cuidado & $\begin{array}{l}\text { Evaluar las relaciones entre el cuidador y la } \\
\text { persona cuidada. } \\
\text { Relaciones de parentesco o de proximidad. } \\
\text { Compartir vivicnda o no. } \\
\text { प Historia del cuidado: tiempo de cuidado, } \\
\text { expectativas de duración, cambios de } \\
\text { residencia... } \\
\text { Otros eventos estresantes en la vida de los } \\
\text { cuidadores. } \\
\text { Caracteristicas del cuidador: edad, estado civil, } \\
\text { sexo, nivel socioeconómico, tipo de trabajo del } \\
\text { cuidador, flexibilidad... }\end{array}$ \\
\hline $\begin{array}{l}\text { Salud física, mental y bienestar } \\
\text { (Efectos del cuidado I) }\end{array}$ & $\begin{array}{ll} & \text { Salud lísica } \\
\square & \text { Salud Mental } \\
\square & \text { Calidad de Vida } \\
\square & \text { Salud percibida } \\
\square & \text { Bienestar percibido } \\
\square & \text { Habilidades del cuidador para realizar las } \\
& \text { actividades básicas de la vida diaria, como } \\
& \text { indicador de habilidad para cuidar a pe rsonas } \\
& \text { mayores. } \\
\square & \text { Satisfacción vital } \\
\square & \text { Ilábitos y estilos de vida }\end{array}$ \\
\hline Carga (Efectos del cuidado II) & $\begin{array}{l}\text { Carga objetiva y Carga subjetiva o percibida } \\
\text { Características del receptor de cuidados en } \\
\text { relación a la carga que producen los délicits de } \\
\text { la persona dependiente. } \\
\text { Tipos de eve ntos estresantes y f recuencia de } \\
\text { aparición. }\end{array}$ \\
\hline Estrategias de Afrontamiento & $\begin{array}{ll} & \text { Solución de problemas } \\
\text { a } & \text { Pasividad. } \\
\text { a } & \text { Evitación. } \\
\text { a } & \text { Instrumentales. } \\
\text { a } & \Lambda \text { fectivas. } \\
\square & \text { Cognitivas } \\
\end{array}$ \\
\hline Apoyo Social & $\begin{array}{ll}\text { a } & \text { Cantidad de relaciones. } \\
\text { a } & \text { Calidad de relaciones } \\
\text { a } & \Lambda \text { poyo espiritual. } \\
\square & \text { Red de relaciones sociales. }\end{array}$ \\
\hline
\end{tabular}

¿Dónde intervenir? Desde cualquier ámbito que tenga contacto con personas con dependencia / familiares.(Hospital, Centros Estancias Diurnas, Residencias, Servicios Sociales Municipales, Asociaciones de familiares, Centros de Salud, etc.)
¿Cuáles son los formatos más frecuentes?

- Elaboración de guías / protocolos.

- Información / orientación /Aseso- 
Tabla 2. Evaluación de los recursos familiares para el ajuste a la discapacidad visual adquirida de un hijo

(Calvo, C., González, J. L, 2003)

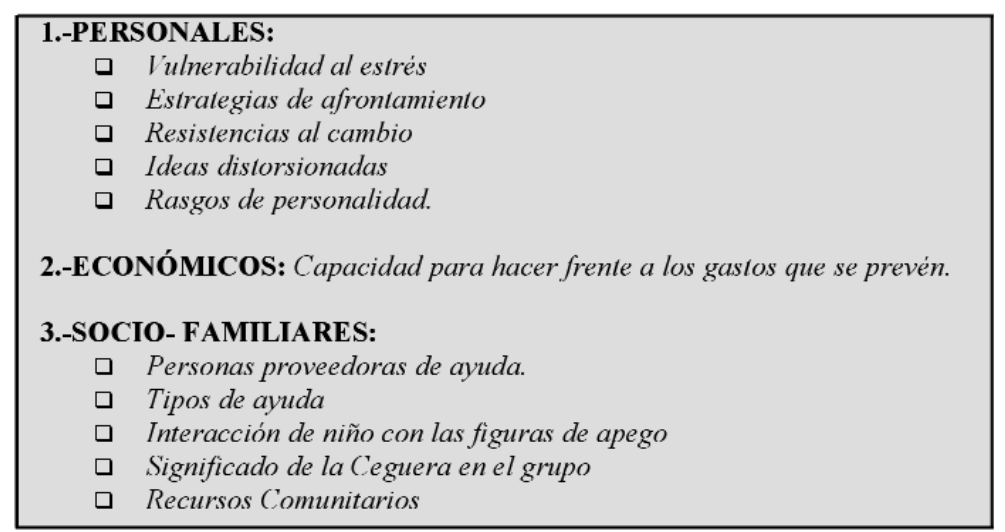

ramiento de profesionales por linea telefónica y/o Internet.

- Intervención psicológica individual: Información / orientación/ asesoramiento / terapia.

\section{- Programa de Ayuda mutua con pro-} fesional. Programa Familiar / Familiar : 1) El profesional ofrece información clínica reestructurando ideas erróneas sobre la enfermedad, discapacidad origen de dependencia; 2) Familiares que han vivido situaciones semejantes ofrecen apoyo en el área emocional y ayuda instrumental.

\section{- Intervención grupal}

- Grupos de Apoyo Social: Intercambio de experiencias entre familiares con situaciones y circunstancias semejantes. Favorece el apoyo social y el intercambio de información.

- Programas Psicoeducativos: Módulo información; Manejo de sentimientos,; Habilidades de afrontamiento. (Ej. Escuelas de padres, grupos psicoeducativos cuidadores ...)
- Instrucción en técnicas o habilidades concretas (comportamientos problemáticos, habilidades de afrontamiento...)

- Programas de intervención clínica con cuidadores: especialmente tratamiento clinico depresión / ansiedad / manejo ira, etc.

- Intervenciones con colectivos muy concretos y necesidades concretas (Ej. Grupos de hermanos de personas con discapacidad cognitivas, grupos terapia duelo tras el fallecimiento de un enfermo de cáncer,...)

\section{EXPERIENCIA PRÁCTICA DE INTERVENCIÓN PSICOLÓGICA CON FAMILIAS DE PERSONAS MAYORES DEPENDIENTES: CENTRO DÍA MUNICIPAL DE PARLA "JOSÉ LUIS SAMPEDRO"}

\section{Contexto de aplicación}

El Centro Día Municipal de Parla (Madrid) "Jose Luis Sampedro" (30 plazas), desde sus comienzos apostó por 
generar un espacio propio para las familias de los usuarios del Centro Día, especialmente orientado al apoyo de los cuidadores/as principales. La intervención psicológica con familias comenzó en el año 2000 y en la actualidad este espacio se ha consolidado procurando ofertar un apoyo continuado, ofreciendo todos los martes 10:30 / 12:00 el apoyo a cuidadores de usuarios del centro / Un Jueves al mes, apoyo a familiares de usuarios que han causado baja. / Intervención anual, con familiares en lista de espera. Los resultados de la intervención psicológica familiar durante estos años han sido muy satisfactorios. El diseño y la organización actual es la siguiente:

- Materiales de apoyo elaborados: Manual "Cuidado del cuidador: El reto e cuidar a nuestros familiares"adaptado de la guía para cuidadores y familiares "Cuando las personas mayores necesitan ayuda" (Izal M., Montorio I., Diaz P., 1997) ; Manual "Habilidades de autocontrol emocional";Cinta / CD Relajación; Elaboración de otros materiales

\section{- Intervención grupal con cuidado-} res familiares usuarios Estancias Diurnas:

- Grupos psicoeducativos "Cuidado del cuidador: El reto de cuidar a nuestros familiares": Desarrollo contenidos Manual.

- Talleres de Entrenamiento grupal en Habilidades de afrontamiento: Taller de relajación; Taller habilidades de autocontrol emocional; Taller habilidades de autocuidado; Otros talleres.

- Grupo de Apoyo: cuidadores familiares.

\section{- Intervención grupal con cuidado}

res familiares en lista de espera Servicio Estancias Diurnas:

- Grupo psicoeducativo " El cuidado del cuidador"

- Intervención grupal / individual apoyo de familiares cuidadores cuyos familiares han causado recientemente bajas en el centro:

- Intervención grupal / individual: Ingreso en Residencia.

- Intervención grupal / individual: Fallecimiento familiar / Duelo.

- Intervención grupal / individual: Empeoramiento Salud.

Taller relajación: Aprender a manejar el estrés

- Familiares y/o cuidadores/as de los usuarios de estancias diurnas. Preferentemente cuidadores principales.

\section{Objetivos de intervención}

A) Objetivos Generales: 1) Promover entre los cuidadores un mejor ajuste a su situación y reducir las consecuencias derivadas de los mismos. 2) Conocer en profundidad la situación de los cuidadores y las necesidades, así como gererar alternativas que contribuyan a mejorarlas. 3) Informar sobre como cuidar mejor al mayor dependiente y proponer hábitos de autocuidado.

B) Objetivos Específicos: 1) Informar sobre qué es y como funciona la respuesta de estrés. 2) Informar sobre la utilidad de las técnicas de relajación para favorecer un autocuidado personal y mejorar la calidad de cuidados a su familiar dependiente. 3) Entrenar al grupo de cuidado- 
res en el uso de técnicas de relajación como instrumento de afrontamiento a las respuestas de estrés. 4) Conseguir respuestas de relajación en el aula y generalizar los resultados a su vida cotidiana. 5) Fomentar el uso de las técnicas de relajación por parte de los cuidadores como instrumento de autocontrol emocional.

\section{Metodologia}

Fase previa:

Detección necesidades: Existencia de varios cuidadores familiares con altos niveles de ansiedad y presencia de sintomas psicosomáticos / trastornos asociados (Ej. Trastornos sueño). Demanda prioritaria por parte de los familiares de formación en estrategias afrontamiento, especialmente técnicas de relajación. La inscripción en el curso se realizó por iniciativa propia. Información telefónica y / o personal ofrecida por la trabajadora social / dirección del centro. Grupo configurado: 10 familiares cuidadores más otros 5 familiares que solicitan beneficiarse de sesiones de manera puntual.

Fase de Ejecución:

El taller constó de 12 sesiones de 90 minutos, distribuidas en dos sesiones semanales del 17 Mayo/24 Junio. El horario $10: 30$ a 12 horas. El curso se realizó en el mismo Centro de Día "José Luis Sampedro" en el que son atendidos sus familiares.

\section{Estructura y Contenidos del taller Relajación}

1. Revisión de deberes para casa: realización de ejercicios fuera del aula.

2. ¿Qué es y cómo funciona el estrés?
3. Detección de fuentes de estrés vinculados al cuidado

4. Alteraciones psicosomáticas frecuentes entre los cuidadores/as.

5. Utilidad de la relajación como técnica de afrontamiento al estrés.

6. La relajación como parte del autocuidado personal del cuidador

7. La relajación como instrumento para la mejora de las relaciones y cuidados de nuestro familiar.

8. Técnicas de relajación: respiración, visualización, entrenamiento autógeno

9. Entrenamiento en el aula de las técnicas de relajación

10. Deberes para casa: realización de ejercicios de relajación fuera del aula.

No se realizó el entrenamiento en técnicas de relajación progresiva al padecer varios miembros del grupo lesiones musculares, problemas en las cervicales y otras articulaciones. En la última sesión se entregó cinta / CD con la grabación de la explicación teórica y los ejercicios de relajación.

\section{Resultados}

1) Datos de asistencia: Asistencia media 71,6\%. El 90\% de las faltas fueron justificadas (realización de gestiones, consultas de médicos bien para ellos/as o para sus familiares). Asistencia $1 / 3$ sesiones de los cuidadores que solicitaron asistir de manera puntual. 
2) Cuestionario cumplimentado por los familiares cuidadores: El cuestionario fue respondido por el $70 \%$ de los asistentes al grupo. Los resultados: Mejora emocional (Mucho (42.8\%) Bastante (57,2\%)). El tiempo dedicado Suficiente (85,7\%). Técnica más útil: Relajación por respiración (100\%); Relajación en aula (100\%) . Relajación en casa(100\%) Métodos más utilizados (57,1\% Respiración, 14,3\% Respiración más Visualización, 28,6\% utiliza todas las técnicas). Relajación en momentos de tensión (100\%). Relajación para el autocontrol emocional ( 71,4\%). Relajación para inducir el sueño $(57,1 \%)$. Relajación para romper ritmo estrés $(85,7 \%)$.

3) Registros / autoinformes niveles relajación en casa: valoración de la ansiedad antes y después de realizar ejercicios de relajación Estos registros fueron entregados por 5 cuidadores familiares: 1) Todos consiguen disminuir su ansiedad con las técnicas de relajación. 2) Dos personas logran alcanzar un nivel de relajación profunda con estas técnicas informando llegar a conseguir niveles de ansiedad 0 . 3) Tres personas logran alcanzar disminuciones en su nivel de ansiedad entre un 40-45 \%. 4) Una persona alcanza una disminución en su nivel de ansiedad del $20 \%$.

4) Otros Resultados: dos cuidadores afirman haber dejado de utilizar medicamentos para la inducción del sueño y otro haber disminuido la presencia de taquicardias (frecuencia, duración).

5) Comentarios a cerca de los datos: Se ha conseguido un alto nivel de cumplimiento de objetivos de intervención. El entrenamiento en grupo ha sido eficaz consiguiendo respuestas de relajación en el aula y en su vida cotidiana. La técnica más usada ha sido la de Respiración, aunque algunos cuidadores han optado por una combinación de varias técnicas. Sería recomendable realizar dos sesiones de seguimiento del mantenimiento de los resultados.

\section{Conclusiones}

$\mathrm{El}$ entrenamiento en técnicas de relajación es eficaz para reducir los niveles de estrés a los que los cuidadores están sometidos, resulta una estrategia útil para el autocontrol emocional, en el afrontamiento de situaciones criticas y en conflictos interpersonales (mayor /familia), favorece hábitos saludables como dormir bien.

\section{CONSIDERACIONES FINALES}

La psicología, desde los años 80 , viene acumulando conocimientos y experiencias prácticas avanzando progresivamente hacia diseños de intervención más eficaces. El desarrollo teórico y práctico en el ámbito de la intervención con familias de personas dependientes, se ha producido de forma paralela en el campo de la discapacidad física, psíquica y sensorial, del envejecimientoy de la enfermedad mental, crónica o terminal. Con la llegada de la ley de la dependencia, se inicia un camino en el que debemos unificar el desarrollo de la psicología. Sería aconsejable favorecer espacios para lograr integrar conocimientos y facilitar intercambios de experiencias a nivel profesional. 


\section{BIBLIOGRAFÍA}

Arranz, P., Barbero, J.J., Barreto, P., Bayés, R. (2003). Intervención emocional en cuidados paliativos: Modelos y protocolos. Ariel Ciencias Médicas.

Braithwaite, V.(1996). Between stressort and outcomes: can we simplify caregiving process variables?. Gerontologist, 36(1), 42-53.

Calvo, G., González, F. (2003). Psicología y ceguera. Manual para la intervención psicológica en el ajuste a la discapacidad visual. Madrid: ONCE.

Haley, W.E., Levine, E.G., Brown, S.L.; Berry, J.W., Hughes, G.H. (1987). Psychological, social and health consequences of caring for a relative with senile dementia. Journal of the American Geritrics Society. 35, 405-411.

INSERSO (1995).Cuidados en la vejez, Madrid. El Apoyo informal. Ministerio de Asuntos Sociales.

IMSERSO (2005). Cuidados a las personas mayores en los hogares españoles. El entorno familiar. Ministerio de Trabajo y Asuntos Sociales.

Izal, M., Montorio, I., Díaz, P. (1997). Cuando las personas mayores necesitan ayuda: Guía para cuidadores y familiares. Ministerio de Trabajo y Asuntos Sociales.

Kahana, E., Biegel, D.E., Wykle, M.L. (1994). Introduction. En E. Kahana; D.E. Biegel y M.L. Wylkle. Family caregiwing across the lifespan. California: Sage.

Lawton, M.P., Brody, E.M., Saperstein, A.R.(1989). A controlled study of respite service for caregivers of Alzheimers patients. The Gerontologist,29(1), 8-16.
Lazarus, R.S., Folkman, S. (1984). Stress, appraisal and coping. New York. Springer Publishing.

Martinez, F., Villaba, C.,García, M. (2001).Revista de Intervención Psicosocial, 10(1), 7-22.

Pearlin, L., Mulland, J., Semple, J., Skaff, M. (1990). Caregiving and the stress process. An overview of concepts and their measures. The Gerontologist, 30,583-594.

Sancho, M., Rodríguez, P. (2002). Envejecimiento y protección social de la dependencia. Veinte años de historia y una mirada al futuro. Revista de Intervención Psicosocial. 10(3).259-275.

Rodríguez, P. (2005). El apoyo informal a las personas mayores en España y la protección social a la dependencia. Del familismo a los derechos de ciudadanía Revista Española de Geriatría y Gerontologia.

Romeis, J.C., (1989). Caregiver strain: Toward an enlarged perspective. Journal of aging and health,1 (2), 188-208.Sage.

Vitaliano, P.P., Russo, J., Young, H.M., Teri L., Maiuro, R.D. (1991). Predictors of burden in spouse caregivers of individuals with alzheimer's disease. Psychol Aging 1991; 6 392-402.

Yanguas, J., Leturia, F. J., Leturia, M., Uriarte, A. (1998). Intervención Psicosocial en Gerontologia: manual práctico. Cáritas.

Zarit, S.H., Reever, K.E., Bach-peterson, J. (1980). Relatives of the impaired aged: correlates of feelings of burden. The Gerontologist, 20, 649655 . 